Acta Crystallographica Section E

Structure Reports

Online

ISSN 1600-5368

\section{Bis(benzyltriethylammonium) hexachloridostannate(IV)}

\section{Ezzatollah Najafi, ${ }^{a}$ Mostafa M. Amini ${ }^{a}$ and Seik Weng $\mathrm{Ng}^{\mathrm{b}}$ *}

${ }^{a}$ Department of Chemistry, General Campus, Shahid Beheshti University, Tehran 1983963113, Iran, and ${ }^{\mathbf{b}}$ Department of Chemistry, University of Malaya, 50603 Kuala Lumpur, Malaysia

Correspondence e-mail: seikweng@um.edu.my

Received 23 September 2010; accepted 24 September 2010

Key indicators: single-crystal X-ray study; $T=295 \mathrm{~K}$; mean $\sigma(\mathrm{C}-\mathrm{C})=0.004 \AA$; $R$ factor $=0.021 ; w R$ factor $=0.059 ;$ data-to-parameter ratio $=23.5$.

The reaction between benzyltriethylammonium chloride and dimethyltin dichloride yields the title salt, $\left[\left(\mathrm{C}_{6} \mathrm{H}_{5} \mathrm{CH}_{2}\right)\right.$ $\left.\left(\mathrm{C}_{2} \mathrm{H}_{5}\right)_{3} \mathrm{~N}\right]_{2}\left[\mathrm{SnCl}_{6}\right]$. The $\mathrm{Sn}^{\text {IV }}$ atom, located on a center of inversion, exists in an octahedral coordination environment. The cation links with the anion via weak $\mathrm{C}-\mathrm{H} \cdots \mathrm{Cl}$ hydrogen bonding.

\section{Related literature}

For bis(tetramethylammonium) hexachloridostannate(IV), see: Furukawa et al. (1982). For bis(tetra- $n$-propylammonium) hexachloridostannate(IV), see: James et al. (1992). For bis(tetraethylammonium) hexachloridostannate(IV), see: Sowa et al. (1981).
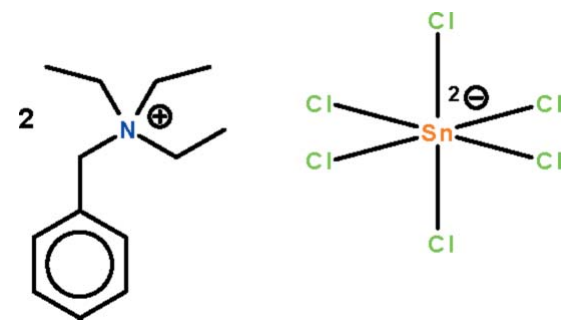

\section{Experimental}

Crystal data

$\begin{array}{ll}\left(\mathrm{C}_{13} \mathrm{H}_{22} \mathrm{~N}\right)_{2}\left[\mathrm{SnCl}_{6}\right] & V=1633.82(15) \AA^{3} \\ M_{r}=716.02 & Z=2 \\ \text { Monoclinic, } P 2_{1} / n & \text { Mo Ko radiation } \\ a=11.2096(6) \AA & \mu=1.29 \mathrm{~mm}^{-1} \\ b=11.2306(6) \AA & T=295 \mathrm{~K} \\ c=12.9796(7) \AA & 0.30 \times 0.20 \times 0.10 \mathrm{~mm}\end{array}$

$\beta=90.872(1)^{\circ}$

\section{Data collection}

Bruker SMART APEX diffractometer

Absorption correction: multi-scan (SADABS; Sheldrick, 1996)

$T_{\min }=0.698, T_{\max }=0.882$

Refinement

$R\left[F^{2}>2 \sigma\left(F^{2}\right)\right]=0.021$

$w R\left(F^{2}\right)=0.059$

$S=1.01$

3756 reflections

15028 measured reflections 3756 independent reflections 3276 reflections with $I>2 \sigma(I)$ $R_{\text {int }}=0.022$

Table 1

Hydrogen-bond geometry $\left(\AA{ }^{\circ}\right)$.

\begin{tabular}{lllll}
\hline$D-\mathrm{H} \cdots A$ & $D-\mathrm{H}$ & $\mathrm{H} \cdots A$ & $D \cdots A$ & $D-\mathrm{H} \cdots A$ \\
\hline $\mathrm{C} 2-\mathrm{H} 2 B \cdots \mathrm{Cl} 1^{\mathrm{i}}$ & 0.96 & 2.74 & $3.685(3)$ & 169
\end{tabular}

Symmetry code: (i) $-x+\frac{1}{2}, y-\frac{1}{2},-z+\frac{3}{2}$.

Data collection: APEX2 (Bruker, 2009); cell refinement: SAINT (Bruker, 2009); data reduction: $S A I N T$; $\operatorname{program}(\mathrm{s})$ used to solve structure: SHELXS97 (Sheldrick, 2008); program(s) used to refine structure: SHELXL97 (Sheldrick, 2008); molecular graphics: $X$ SEED (Barbour, 2001); software used to prepare material for publication: publCIF (Westrip, 2010).

We thank Shahid Beheshti University and the University of Malaya for supporting this study.

Supplementary data and figures for this paper are available from the IUCr electronic archives (Reference: XU5035).

\section{References}

Barbour, L. J. (2001). J. Supramol. Chem. 1, 189-191.

Bruker (2009). APEX2 and SAINT. Bruker AXS Inc., Madison, Wisconsin, USA.

Furukawa, Y., Prabhumirashi, L. S., Ikeda, R. \& Nakamura, D. (1982). Bull. Chem. Soc. Jpn, 55, 995-998.

James, M. A., Knop, O. \& Cameron, T. S. (1992). Can. J. Chem. 70, 1795-1821. Sheldrick, G. M. (1996). SADABS. University of Göttingen, Germany. Sheldrick, G. M. (2008). Acta Cryst. A64, 112-122.

Sowa, H., Druck, U. \& Kutoglu, A. (1981). Cryst. Struct. Commun. 10, 699-702. Westrip, S. P. (2010). J. Appl. Cryst. 43, 920-925. 


\section{supporting information}

Acta Cryst. (2010). E66, m1334 [doi:10.1107/S1600536810038122]

\section{Bis(benzyltriethylammonium) hexachloridostannate(IV)}

\section{Ezzatollah Najafi, Mostafa M. Amini and Seik Weng Ng}

\section{S1. Comment}

The reaction of dimethyltin dichloride with ammonium halides sometimes leads to tin-carbon cleave to result in the formation of a hexahalogenostannate. Tin-methyl cleavage was noted in the reaction of dimethyltin dichloride with and benzyltriethylammonium chloride; the resulting the title salt (Scheme I, Fig. 1) consists of ammonium cations and hexachloridostannate anions. The reported ammonium hexachloridostannates all have symmetrically substituted ammonium cations.

\section{S2. Experimental}

Dimethyltin(IV) dichloride $(0.219 \mathrm{~g}, 1 \mathrm{mmol})$ and benzyltriethylammonium chloride $(0.455 \mathrm{~g}, 2 \mathrm{mmol})$ were dissolved in methanol and the solution kept at $333 \mathrm{~K}$. Crystals were isolated after several days; m.p. 452-454 K.

\section{S3. Refinement}

Hydrogen atoms were placed in calculated positions $(\mathrm{C}-\mathrm{H} 0.93-0.97 \AA)$ and were included in the refinement in the riding model approximation, with $U(\mathrm{H})$ set to $1.2-1.5 U_{\mathrm{eq}}(\mathrm{C})$.
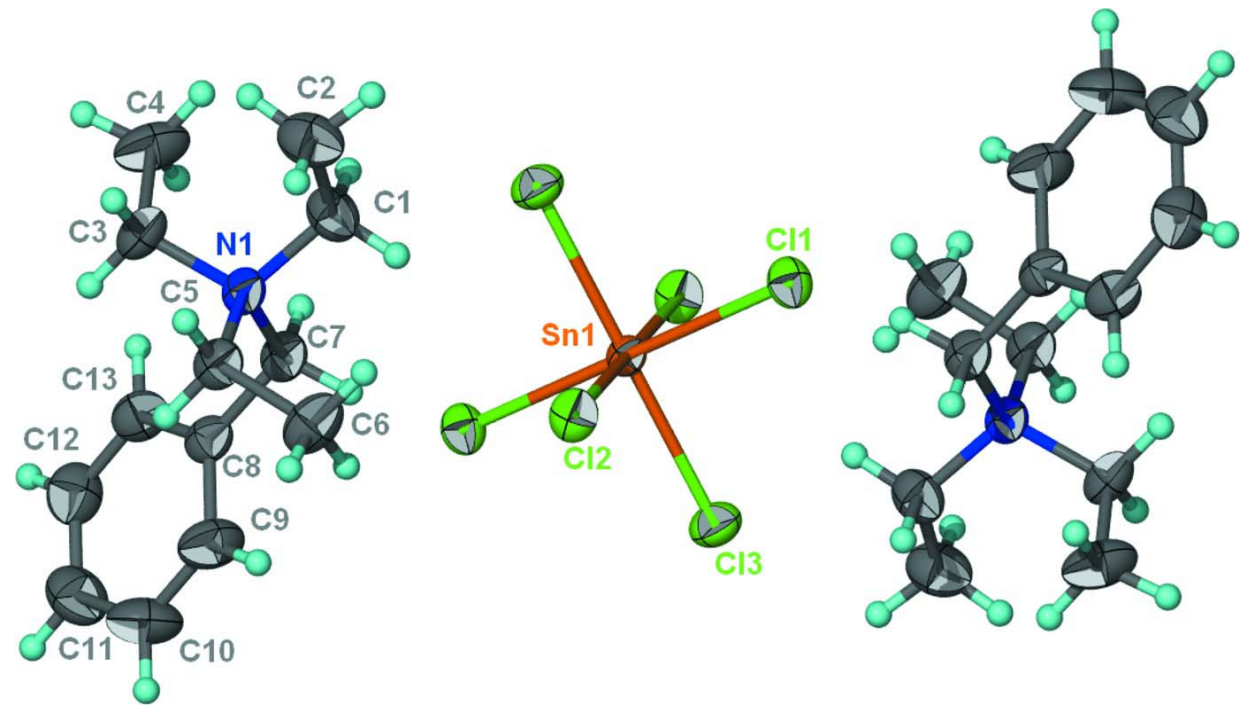

\section{Figure 1}

Thermal ellipsoid plot (Barbour, 2001) of the $2\left(\mathrm{C}_{6} \mathrm{H}_{5} \mathrm{CH}_{2}\right)\left(\mathrm{C}_{2} \mathrm{H}_{5}\right)_{3} \mathrm{~N}^{+} \mathrm{SnCl}_{6}{ }^{2-}$ salt at the $50 \%$ probability level. Hydrogen atoms are drawn as spheres of arbitrary radius. 


\section{Bis(benzyltriethylammonium) hexachloridostannate(IV)}

Crystal data

$\left(\mathrm{C}_{13} \mathrm{H}_{22} \mathrm{~N}\right)_{2}\left[\mathrm{SnCl}_{6}\right]$

$M_{r}=716.02$

Monoclinic, $P 2_{1} / n$

Hall symbol: -P 2 yn

$a=11.2096(6) \AA$

$b=11.2306(6) \AA$

$c=12.9796(7) \AA$

$\beta=90.872(1)^{\circ}$

$V=1633.82(15) \AA^{3}$

$Z=2$

\section{Data collection}

Bruker SMART APEX

diffractometer

Radiation source: fine-focus sealed tube

Graphite monochromator

$\omega$ scans

Absorption correction: multi-scan

(SADABS; Sheldrick, 1996)

$T_{\min }=0.698, T_{\max }=0.882$

Refinement

Refinement on $F^{2}$

Least-squares matrix: full

$R\left[F^{2}>2 \sigma\left(F^{2}\right)\right]=0.021$

$w R\left(F^{2}\right)=0.059$

$S=1.01$

3756 reflections

160 parameters

0 restraints

Primary atom site location: structure-invariant direct methods
$F(000)=732$

$D_{\mathrm{x}}=1.455 \mathrm{Mg} \mathrm{m}^{-3}$

Mo $K \alpha$ radiation, $\lambda=0.71073 \AA$

Cell parameters from 8245 reflections

$\theta=2.4-28.2^{\circ}$

$\mu=1.29 \mathrm{~mm}^{-1}$

$T=295 \mathrm{~K}$

Prism, colorless

$0.30 \times 0.20 \times 0.10 \mathrm{~mm}$

15028 measured reflections

3756 independent reflections

3276 reflections with $I>2 \sigma(I)$

$R_{\text {int }}=0.022$

$\theta_{\text {max }}=27.5^{\circ}, \theta_{\min }=2.4^{\circ}$

$h=-14 \rightarrow 13$

$k=-14 \rightarrow 14$

$l=-16 \rightarrow 16$

Secondary atom site location: difference Fourier map

Hydrogen site location: inferred from

neighbouring sites

$\mathrm{H}$-atom parameters constrained

$w=1 /\left[\sigma^{2}\left(F_{0}^{2}\right)+(0.032 P)^{2}+0.4848 P\right]$

where $P=\left(F_{\mathrm{o}}^{2}+2 F_{\mathrm{c}}^{2}\right) / 3$

$(\Delta / \sigma)_{\max }=0.001$

$\Delta \rho_{\max }=0.41 \mathrm{e} \AA^{-3}$

$\Delta \rho_{\min }=-0.39$ e $\AA^{-3}$

Fractional atomic coordinates and isotropic or equivalent isotropic displacement parameters $\left(\hat{A}^{2}\right)$

\begin{tabular}{lllll}
\hline & $x$ & $y$ & $z$ & $U_{\text {iso }} / U_{\text {eq }}$ \\
\hline Sn1 & 0.5000 & 0.5000 & 0.5000 & $0.03041(6)$ \\
C11 & $0.53546(4)$ & $0.67877(4)$ & $0.60050(3)$ & $0.04330(11)$ \\
C12 & $0.45054(4)$ & $0.39111(4)$ & $0.65796(3)$ & $0.04483(12)$ \\
C13 & $0.70731(4)$ & $0.44472(5)$ & $0.52383(4)$ & $0.04963(13)$ \\
N1 & $0.10908(13)$ & $0.22025(14)$ & $0.56788(12)$ & $0.0390(3)$ \\
C1 & $0.1030(2)$ & $0.3475(2)$ & $0.60638(18)$ & $0.0534(5)$ \\
H1A & 0.1762 & 0.3649 & 0.6443 & $0.064^{*}$ \\
H1B & 0.0997 & 0.4002 & 0.5472 & $0.064^{*}$ \\
C2 & $-0.0015(2)$ & $0.3758(2)$ & $0.6748(2)$ & $0.0659(7)$ \\
H2A & 0.0027 & 0.4576 & 0.6958 & $0.099^{*}$ \\
H2B & 0.0013 & 0.3254 & 0.7345 & $0.099^{*}$ \\
H2C & -0.0748 & 0.3622 & 0.6373 & $0.099^{*}$ \\
C3 & $-0.00149(17)$ & $0.1864(2)$ & $0.50650(16)$ & $0.0519(5)$
\end{tabular}




$\begin{array}{lllll}\text { H3A } & -0.0680 & 0.1816 & 0.5533 & 0.062^{*} \\ \text { H3B } & 0.0103 & 0.1075 & 0.4779 & 0.062^{*} \\ \text { C4 } & -0.0354(2) & 0.2704(3) & 0.41919(19) & 0.0744(8) \\ \text { H4A } & -0.1066 & 0.2421 & 0.3853 & 0.112^{*} \\ \text { H4B } & 0.0283 & 0.2736 & 0.3707 & 0.112^{*} \\ \text { H4C } & -0.0492 & 0.3486 & 0.4464 & 0.112^{*} \\ \text { C5 } & 0.11816(18) & 0.13332(19) & 0.65726(15) & 0.0460(5) \\ \text { H5A } & 0.1295 & 0.0539 & 0.6296 & 0.055^{*} \\ \text { H5B } & 0.0429 & 0.1336 & 0.6932 & 0.055^{*} \\ \text { C6 } & 0.2178(2) & 0.1584(3) & 0.73500(17) & 0.0627(6) \\ \text { H6A } & 0.2181 & 0.0979 & 0.7873 & 0.094^{*} \\ \text { H6B } & 0.2051 & 0.2348 & 0.7662 & 0.094^{*} \\ \text { H6C } & 0.2930 & 0.1583 & 0.7007 & 0.094^{*} \\ \text { C7 } & 0.22048(17) & 0.21310(18) & 0.50080(15) & 0.0436(4) \\ \text { H7A } & 0.2122 & 0.2710 & 0.4457 & 0.052^{*} \\ \text { H7B } & 0.2891 & 0.2363 & 0.5426 & 0.052^{*} \\ \text { C8 } & 0.24596(17) & 0.09404(19) & 0.45360(15) & 0.0429(4) \\ \text { C9 } & 0.3185(3) & 0.0112(2) & 0.5036(2) & 0.0660(7) \\ \text { H9 } & 0.3492 & 0.0284 & 0.5689 & 0.079^{*} \\ \text { C10 } & 0.3458(3) & -0.0965(3) & 0.4577(2) & 0.0798(8) \\ \text { H10 } & 0.3933 & -0.1515 & 0.4927 & 0.096^{*} \\ \text { C11 } & 0.3032(3) & -0.1225(2) & 0.3612(2) & 0.0713(7) \\ \text { H11 } & 0.3217 & -0.1949 & 0.3306 & 0.086^{*} \\ \text { C12 } & 0.2334(2) & -0.0415(3) & 0.3098(2) & 0.0632(6) \\ \text { H12 } & 0.2049 & -0.0587 & 0.2438 & 0.076^{*} \\ \text { C13 } & 0.2049(2) & 0.0659(2) & 0.35541(16) & 0.0524(5) \\ \text { H13 } & 0.1574 & 0.1202 & 0.3195 & 0.063^{*}\end{array}$

Atomic displacement parameters $\left(\AA^{2}\right)$

\begin{tabular}{lllllll}
\hline & $U^{11}$ & $U^{22}$ & $U^{33}$ & $U^{12}$ & $U^{13}$ & $U^{23}$ \\
\hline Sn1 & $0.02733(9)$ & $0.03422(10)$ & $0.02977(9)$ & $-0.00209(6)$ & $0.00331(6)$ & $0.00222(6)$ \\
C11 & $0.0462(3)$ & $0.0419(2)$ & $0.0420(2)$ & $-0.0050(2)$ & $0.00723(19)$ & $-0.00544(19)$ \\
C12 & $0.0483(3)$ & $0.0508(3)$ & $0.0354(2)$ & $-0.0082(2)$ & $0.00231(18)$ & $0.00960(19)$ \\
C13 & $0.0344(2)$ & $0.0550(3)$ & $0.0595(3)$ & $0.0041(2)$ & $0.0010(2)$ & $0.0053(2)$ \\
N1 & $0.0344(8)$ & $0.0408(8)$ & $0.0418(8)$ & $-0.0066(6)$ & $0.0041(6)$ & $0.0081(7)$ \\
C1 & $0.0606(14)$ & $0.0438(11)$ & $0.0561(12)$ & $-0.0041(10)$ & $0.0122(10)$ & $0.0042(9)$ \\
C2 & $0.0721(16)$ & $0.0610(15)$ & $0.0650(15)$ & $0.0120(13)$ & $0.0182(12)$ & $0.0052(12)$ \\
C3 & $0.0346(10)$ & $0.0675(15)$ & $0.0536(12)$ & $-0.0047(9)$ & $-0.0041(9)$ & $0.0039(10)$ \\
C4 & $0.0640(16)$ & $0.101(2)$ & $0.0578(14)$ & $0.0305(15)$ & $-0.0091(12)$ & $0.0077(14)$ \\
C5 & $0.0429(11)$ & $0.0497(11)$ & $0.0454(10)$ & $-0.0070(9)$ & $0.0037(8)$ & $0.0154(9)$ \\
C6 & $0.0511(13)$ & $0.0902(19)$ & $0.0467(12)$ & $-0.0049(12)$ & $-0.0053(10)$ & $0.0124(12)$ \\
C7 & $0.0375(10)$ & $0.0483(11)$ & $0.0453(10)$ & $-0.0073(8)$ & $0.0074(8)$ & $0.0075(8)$ \\
C8 & $0.0348(10)$ & $0.0498(11)$ & $0.0443(10)$ & $-0.0025(8)$ & $0.0033(8)$ & $0.0071(8)$ \\
C9 & $0.0628(16)$ & $0.0793(19)$ & $0.0554(14)$ & $0.0197(13)$ & $-0.0120(12)$ & $0.0016(12)$ \\
C10 & $0.081(2)$ & $0.0746(19)$ & $0.084(2)$ & $0.0352(16)$ & $-0.0011(15)$ & $0.0087(15)$ \\
C11 & $0.0777(18)$ & $0.0575(15)$ & $0.0793(18)$ & $0.0054(13)$ & $0.0182(14)$ & $-0.0086(13)$ \\
C12 & $0.0640(15)$ & $0.0723(16)$ & $0.0535(13)$ & $-0.0050(13)$ & $0.0044(11)$ & $-0.0103(12)$
\end{tabular}




$\begin{array}{lllllll}\mathrm{C} 13 & 0.0502(12) & 0.0615(14) & 0.0453(11) & 0.0037(10) & -0.0008(9) & 0.0070(10)\end{array}$

Geometric parameters $(\AA, \stackrel{o}{)}$

\begin{tabular}{|c|c|c|c|}
\hline $\mathrm{Sn} 1-\mathrm{Cl}^{\mathrm{i}}$ & $2.4207(5)$ & $\mathrm{C} 4-\mathrm{H} 4 \mathrm{C}$ & 0.9600 \\
\hline $\mathrm{Sn} 1-\mathrm{Cl} 3$ & $2.4207(5)$ & $\mathrm{C} 5-\mathrm{C} 6$ & $1.520(3)$ \\
\hline $\mathrm{Sn} 1-\mathrm{Cl1}$ & $2.4237(5)$ & $\mathrm{C} 5-\mathrm{H} 5 \mathrm{~A}$ & 0.9700 \\
\hline $\mathrm{Sn} 1-\mathrm{Cl1}^{\mathrm{i}}$ & $2.4237(5)$ & $\mathrm{C} 5-\mathrm{H} 5 \mathrm{~B}$ & 0.9700 \\
\hline $\mathrm{Sn} 1-\mathrm{Cl} 2^{\mathrm{i}}$ & $2.4579(4)$ & C6-H6A & 0.9600 \\
\hline $\mathrm{Sn} 1-\mathrm{Cl} 2$ & $2.4579(4)$ & C6-H6B & 0.9600 \\
\hline $\mathrm{N} 1-\mathrm{C} 3$ & $1.512(2)$ & $\mathrm{C} 6-\mathrm{H} 6 \mathrm{C}$ & 0.9600 \\
\hline $\mathrm{N} 1-\mathrm{C} 1$ & $1.515(3)$ & $\mathrm{C} 7-\mathrm{C} 8$ & $1.500(3)$ \\
\hline $\mathrm{N} 1-\mathrm{C} 5$ & $1.518(2)$ & C7-H7A & 0.9700 \\
\hline $\mathrm{N} 1-\mathrm{C} 7$ & $1.535(2)$ & $\mathrm{C} 7-\mathrm{H} 7 \mathrm{~B}$ & 0.9700 \\
\hline $\mathrm{C} 1-\mathrm{C} 2$ & $1.515(3)$ & $\mathrm{C} 8-\mathrm{C} 13$ & $1.385(3)$ \\
\hline $\mathrm{C} 1-\mathrm{H} 1 \mathrm{~A}$ & 0.9700 & $\mathrm{C} 8-\mathrm{C} 9$ & $1.390(3)$ \\
\hline $\mathrm{C} 1-\mathrm{H} 1 \mathrm{~B}$ & 0.9700 & $\mathrm{C} 9-\mathrm{C} 10$ & $1.386(4)$ \\
\hline $\mathrm{C} 2-\mathrm{H} 2 \mathrm{~A}$ & 0.9600 & $\mathrm{C} 9-\mathrm{H} 9$ & 0.9300 \\
\hline $\mathrm{C} 2-\mathrm{H} 2 \mathrm{~B}$ & 0.9600 & $\mathrm{C} 10-\mathrm{C} 11$ & $1.364(4)$ \\
\hline $\mathrm{C} 2-\mathrm{H} 2 \mathrm{C}$ & 0.9600 & $\mathrm{C} 10-\mathrm{H} 10$ & 0.9300 \\
\hline $\mathrm{C} 3-\mathrm{C} 4$ & $1.519(3)$ & $\mathrm{C} 11-\mathrm{C} 12$ & $1.367(4)$ \\
\hline $\mathrm{C} 3-\mathrm{H} 3 \mathrm{~A}$ & 0.9700 & $\mathrm{C} 11-\mathrm{H} 11$ & 0.9300 \\
\hline $\mathrm{C} 3-\mathrm{H} 3 \mathrm{~B}$ & 0.9700 & $\mathrm{C} 12-\mathrm{C} 13$ & $1.384(4)$ \\
\hline $\mathrm{C} 4-\mathrm{H} 4 \mathrm{~A}$ & 0.9600 & $\mathrm{C} 12-\mathrm{H} 12$ & 0.9300 \\
\hline $\mathrm{C} 4-\mathrm{H} 4 \mathrm{~B}$ & 0.9600 & $\mathrm{C} 13-\mathrm{H} 13$ & 0.9300 \\
\hline $\mathrm{Cl} 3^{\mathrm{i}}-\mathrm{Sn} 1-\mathrm{Cl} 3$ & 180.0 & $\mathrm{H} 4 \mathrm{~A}-\mathrm{C} 4-\mathrm{H} 4 \mathrm{~B}$ & 109.5 \\
\hline $\mathrm{Cl} 3^{\mathrm{i}}-\mathrm{Sn} 1-\mathrm{Cl} 1$ & $90.320(18)$ & $\mathrm{C} 3-\mathrm{C} 4-\mathrm{H} 4 \mathrm{C}$ & 109.5 \\
\hline $\mathrm{Cl} 3-\mathrm{Sn} 1-\mathrm{Cl} 1$ & $89.680(18)$ & $\mathrm{H} 4 \mathrm{~A}-\mathrm{C} 4-\mathrm{H} 4 \mathrm{C}$ & 109.5 \\
\hline $\mathrm{Cl} 3^{\mathrm{i}}-\mathrm{Sn} 1-\mathrm{Cl} 1^{\mathrm{i}}$ & $89.680(18)$ & $\mathrm{H} 4 \mathrm{~B}-\mathrm{C} 4-\mathrm{H} 4 \mathrm{C}$ & 109.5 \\
\hline $\mathrm{Cl} 3-\mathrm{Sn} 1-\mathrm{Cl}^{\mathrm{i}}$ & $90.320(18)$ & $\mathrm{N} 1-\mathrm{C} 5-\mathrm{C} 6$ & $115.36(17)$ \\
\hline $\mathrm{C} 11-\mathrm{Sn} 1-\mathrm{Cl1}^{\mathrm{i}}$ & 180.0 & $\mathrm{~N} 1-\mathrm{C} 5-\mathrm{H} 5 \mathrm{~A}$ & 108.4 \\
\hline 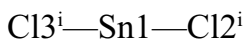 & $89.662(17)$ & $\mathrm{C} 6-\mathrm{C} 5-\mathrm{H} 5 \mathrm{~A}$ & 108.4 \\
\hline $\mathrm{Cl} 3-\mathrm{Sn} 1-\mathrm{Cl} 2^{\mathrm{i}}$ & $90.338(17)$ & $\mathrm{N} 1-\mathrm{C} 5-\mathrm{H} 5 \mathrm{~B}$ & 108.4 \\
\hline $\mathrm{C} 11-\mathrm{Sn} 1-\mathrm{C} 12^{\mathrm{i}}$ & $89.974(17)$ & $\mathrm{C} 6-\mathrm{C} 5-\mathrm{H} 5 \mathrm{~B}$ & 108.4 \\
\hline $\mathrm{Cl} 1^{\mathrm{i}}-\mathrm{Sn} 1-\mathrm{Cl} 2^{\mathrm{i}}$ & $90.026(17)$ & $\mathrm{H} 5 \mathrm{~A}-\mathrm{C} 5-\mathrm{H} 5 \mathrm{~B}$ & 107.5 \\
\hline 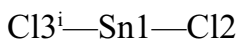 & $90.338(17)$ & $\mathrm{C} 5-\mathrm{C} 6-\mathrm{H} 6 \mathrm{~A}$ & 109.5 \\
\hline $\mathrm{Cl} 3-\mathrm{Sn} 1-\mathrm{Cl} 2$ & $89.662(17)$ & $\mathrm{C} 5-\mathrm{C} 6-\mathrm{H} 6 \mathrm{~B}$ & 109.5 \\
\hline $\mathrm{C} 11-\mathrm{Sn} 1-\mathrm{Cl} 2$ & $90.026(17)$ & $\mathrm{H} 6 \mathrm{~A}-\mathrm{C} 6-\mathrm{H} 6 \mathrm{~B}$ & 109.5 \\
\hline $\mathrm{Cl} 1{ }^{\mathrm{i}}-\mathrm{Sn} 1-\mathrm{Cl} 2$ & 89.974 (17) & $\mathrm{C} 5-\mathrm{C} 6-\mathrm{H} 6 \mathrm{C}$ & 109.5 \\
\hline $\mathrm{Cl} 2^{\mathrm{i}}-\mathrm{Sn} 1-\mathrm{Cl} 2$ & $180.00(2)$ & $\mathrm{H} 6 \mathrm{~A}-\mathrm{C} 6-\mathrm{H} 6 \mathrm{C}$ & 109.5 \\
\hline $\mathrm{C} 3-\mathrm{N} 1-\mathrm{C} 1$ & $111.75(17)$ & $\mathrm{H} 6 \mathrm{~B}-\mathrm{C} 6-\mathrm{H} 6 \mathrm{C}$ & 109.5 \\
\hline $\mathrm{C} 3-\mathrm{N} 1-\mathrm{C} 5$ & $106.60(15)$ & $\mathrm{C} 8-\mathrm{C} 7-\mathrm{N} 1$ & $116.09(15)$ \\
\hline $\mathrm{C} 1-\mathrm{N} 1-\mathrm{C} 5$ & $110.92(16)$ & $\mathrm{C} 8-\mathrm{C} 7-\mathrm{H} 7 \mathrm{~A}$ & 108.3 \\
\hline $\mathrm{C} 3-\mathrm{N} 1-\mathrm{C} 7$ & $110.82(15)$ & $\mathrm{N} 1-\mathrm{C} 7-\mathrm{H} 7 \mathrm{~A}$ & 108.3 \\
\hline $\mathrm{C} 1-\mathrm{N} 1-\mathrm{C} 7$ & $106.10(14)$ & $\mathrm{C} 8-\mathrm{C} 7-\mathrm{H} 7 \mathrm{~B}$ & 108.3 \\
\hline $\mathrm{C} 5-\mathrm{N} 1-\mathrm{C} 7$ & $110.73(15)$ & $\mathrm{N} 1-\mathrm{C} 7-\mathrm{H} 7 \mathrm{~B}$ & 108.3 \\
\hline $\mathrm{C} 2-\mathrm{C} 1-\mathrm{N} 1$ & $115.49(18)$ & $\mathrm{H} 7 \mathrm{~A}-\mathrm{C} 7-\mathrm{H} 7 \mathrm{~B}$ & 107.4 \\
\hline
\end{tabular}




\begin{tabular}{|c|c|c|c|}
\hline $\mathrm{C} 2-\mathrm{C} 1-\mathrm{H} 1 \mathrm{~A}$ & 108.4 & $\mathrm{C} 13-\mathrm{C} 8-\mathrm{C} 9$ & $117.5(2)$ \\
\hline $\mathrm{N} 1-\mathrm{C} 1-\mathrm{H} 1 \mathrm{~A}$ & 108.4 & $\mathrm{C} 13-\mathrm{C} 8-\mathrm{C} 7$ & $121.09(19)$ \\
\hline $\mathrm{C} 2-\mathrm{C} 1-\mathrm{H} 1 \mathrm{~B}$ & 108.4 & $\mathrm{C} 9-\mathrm{C} 8-\mathrm{C} 7$ & $121.3(2)$ \\
\hline $\mathrm{N} 1-\mathrm{C} 1-\mathrm{H} 1 \mathrm{~B}$ & 108.4 & $\mathrm{C} 10-\mathrm{C} 9-\mathrm{C} 8$ & $121.0(2)$ \\
\hline $\mathrm{H} 1 \mathrm{~A}-\mathrm{C} 1-\mathrm{H} 1 \mathrm{~B}$ & 107.5 & $\mathrm{C} 10-\mathrm{C} 9-\mathrm{H} 9$ & 119.5 \\
\hline $\mathrm{C} 1-\mathrm{C} 2-\mathrm{H} 2 \mathrm{~A}$ & 109.5 & $\mathrm{C} 8-\mathrm{C} 9-\mathrm{H} 9$ & 119.5 \\
\hline $\mathrm{C} 1-\mathrm{C} 2-\mathrm{H} 2 \mathrm{~B}$ & 109.5 & $\mathrm{C} 11-\mathrm{C} 10-\mathrm{C} 9$ & $120.3(3)$ \\
\hline $\mathrm{H} 2 \mathrm{~A}-\mathrm{C} 2-\mathrm{H} 2 \mathrm{~B}$ & 109.5 & $\mathrm{C} 11-\mathrm{C} 10-\mathrm{H} 10$ & 119.8 \\
\hline $\mathrm{C} 1-\mathrm{C} 2-\mathrm{H} 2 \mathrm{C}$ & 109.5 & $\mathrm{C} 9-\mathrm{C} 10-\mathrm{H} 10$ & 119.8 \\
\hline $\mathrm{H} 2 \mathrm{~A}-\mathrm{C} 2-\mathrm{H} 2 \mathrm{C}$ & 109.5 & $\mathrm{C} 10-\mathrm{C} 11-\mathrm{C} 12$ & $119.7(3)$ \\
\hline $\mathrm{H} 2 \mathrm{~B}-\mathrm{C} 2-\mathrm{H} 2 \mathrm{C}$ & 109.5 & $\mathrm{C} 10-\mathrm{C} 11-\mathrm{H} 11$ & 120.1 \\
\hline $\mathrm{N} 1-\mathrm{C} 3-\mathrm{C} 4$ & $115.5(2)$ & $\mathrm{C} 12-\mathrm{C} 11-\mathrm{H} 11$ & 120.1 \\
\hline $\mathrm{N} 1-\mathrm{C} 3-\mathrm{H} 3 \mathrm{~A}$ & 108.4 & $\mathrm{C} 11-\mathrm{C} 12-\mathrm{C} 13$ & $120.4(2)$ \\
\hline $\mathrm{C} 4-\mathrm{C} 3-\mathrm{H} 3 \mathrm{~A}$ & 108.4 & $\mathrm{C} 11-\mathrm{C} 12-\mathrm{H} 12$ & 119.8 \\
\hline $\mathrm{N} 1-\mathrm{C} 3-\mathrm{H} 3 \mathrm{~B}$ & 108.4 & $\mathrm{C} 13-\mathrm{C} 12-\mathrm{H} 12$ & 119.8 \\
\hline $\mathrm{C} 4-\mathrm{C} 3-\mathrm{H} 3 \mathrm{~B}$ & 108.4 & $\mathrm{C} 8-\mathrm{C} 13-\mathrm{C} 12$ & $121.1(2)$ \\
\hline $\mathrm{H} 3 \mathrm{~A}-\mathrm{C} 3-\mathrm{H} 3 \mathrm{~B}$ & 107.5 & $\mathrm{C} 8-\mathrm{C} 13-\mathrm{H} 13$ & 119.4 \\
\hline $\mathrm{C} 3-\mathrm{C} 4-\mathrm{H} 4 \mathrm{~A}$ & 109.5 & $\mathrm{C} 12-\mathrm{C} 13-\mathrm{H} 13$ & 119.4 \\
\hline $\mathrm{C} 3-\mathrm{C} 4-\mathrm{H} 4 \mathrm{~B}$ & 109.5 & & \\
\hline $\mathrm{C} 3-\mathrm{N} 1-\mathrm{C} 1-\mathrm{C} 2$ & $58.9(2)$ & $\mathrm{C} 5-\mathrm{N} 1-\mathrm{C} 7-\mathrm{C} 8$ & $59.4(2)$ \\
\hline $\mathrm{C} 5-\mathrm{N} 1-\mathrm{C} 1-\mathrm{C} 2$ & $-59.9(2)$ & $\mathrm{N} 1-\mathrm{C} 7-\mathrm{C} 8-\mathrm{C} 13$ & $93.4(2)$ \\
\hline $\mathrm{C} 7-\mathrm{N} 1-\mathrm{C} 1-\mathrm{C} 2$ & $179.8(2)$ & $\mathrm{N} 1-\mathrm{C} 7-\mathrm{C} 8-\mathrm{C} 9$ & $-91.3(3)$ \\
\hline $\mathrm{C} 1-\mathrm{N} 1-\mathrm{C} 3-\mathrm{C} 4$ & $52.4(2)$ & $\mathrm{C} 13-\mathrm{C} 8-\mathrm{C} 9-\mathrm{C} 10$ & $-1.7(4)$ \\
\hline $\mathrm{C} 5-\mathrm{N} 1-\mathrm{C} 3-\mathrm{C} 4$ & $173.76(19)$ & $\mathrm{C} 7-\mathrm{C} 8-\mathrm{C} 9-\mathrm{C} 10$ & $-177.2(3)$ \\
\hline $\mathrm{C} 7-\mathrm{N} 1-\mathrm{C} 3-\mathrm{C} 4$ & $-65.7(2)$ & $\mathrm{C} 8-\mathrm{C} 9-\mathrm{C} 10-\mathrm{C} 11$ & $1.1(5)$ \\
\hline $\mathrm{C} 3-\mathrm{N} 1-\mathrm{C} 5-\mathrm{C} 6$ & $-174.68(19)$ & $\mathrm{C} 9-\mathrm{C} 10-\mathrm{C} 11-\mathrm{C} 12$ & $0.0(5)$ \\
\hline $\mathrm{C} 1-\mathrm{N} 1-\mathrm{C} 5-\mathrm{C} 6$ & $-52.8(2)$ & $\mathrm{C} 10-\mathrm{C} 11-\mathrm{C} 12-\mathrm{C} 13$ & $-0.5(4)$ \\
\hline $\mathrm{C} 7-\mathrm{N} 1-\mathrm{C} 5-\mathrm{C} 6$ & $64.7(2)$ & $\mathrm{C} 9-\mathrm{C} 8-\mathrm{C} 13-\mathrm{C} 12$ & $1.2(3)$ \\
\hline $\mathrm{C} 3-\mathrm{N} 1-\mathrm{C} 7-\mathrm{C} 8$ & $-58.7(2)$ & $\mathrm{C} 7-\mathrm{C} 8-\mathrm{C} 13-\mathrm{C} 12$ & $176.7(2)$ \\
\hline $\mathrm{C} 1-\mathrm{N} 1-\mathrm{C} 7-\mathrm{C} 8$ & $179.82(17)$ & $\mathrm{C} 11-\mathrm{C} 12-\mathrm{C} 13-\mathrm{C} 8$ & $-0.1(4)$ \\
\hline
\end{tabular}

Symmetry code: (i) $-x+1,-y+1,-z+1$.

Hydrogen-bond geometry $\left(\AA,{ }^{\circ}\right)$

\begin{tabular}{lllll}
\hline$D-\mathrm{H} \cdots A$ & $D-\mathrm{H}$ & $\mathrm{H} \cdots A$ & $D \cdots A$ & $D-\mathrm{H} \cdots A$ \\
\hline $\mathrm{C} 2-\mathrm{H} 2 B \cdots \mathrm{C} 11^{1 \mathrm{ii}}$ & 0.96 & 2.74 & $3.685(3)$ & 169 \\
\hline
\end{tabular}

Symmetry code: (ii) $-x+1 / 2, y-1 / 2,-z+3 / 2$. 